\title{
Characterization of CR-Lightlike-Warped Product of Indefinite Kaehler Manifolds
}

\author{
Rachna Rani, ${ }^{1}$ Rakesh Kumar, ${ }^{2}$ and R. K. Nagaich ${ }^{3}$ \\ ${ }^{1}$ University College, Moonak 148033, India \\ ${ }^{2}$ University College of Engineering, Punjabi University, Patiala 147002, India \\ ${ }^{3}$ Department of Mathematics, Punjabi University, Patiala 147002, India
}

Correspondence should be addressed to Rakesh Kumar, dr_rk37c@yahoo.co.in

Received 26 September 2011; Accepted 19 October 2011

Academic Editors: B. Gato Rivera, D. Gepner, and F. Sugino

Copyright @ 2012 Rachna Rani et al. This is an open access article distributed under the Creative Commons Attribution License, which permits unrestricted use, distribution, and reproduction in any medium, provided the original work is properly cited.

In this paper, we prove that there does not exist a warped product CR-lightlike submanifold in the form $M=N_{\perp} \times{ }_{\lambda} N_{T}$ other than CR-lightlike product in an indefinite Kaehler manifold. We also obtain some characterizations for a CR-lightlike submanifold to be locally a CR-lightlike warped product.

\section{Introduction}

The general theory of Cauchy-Riemann (CR-) submanifolds of Kaehler manifolds, being generalization of holomorphic and totally real submanifolds of Kaehler manifolds, was initiated in Bejancu [1] and has been further developed in [2-4] and others. Later on, Duggal and Bejancu [5] introduced a new class called CR-lightlike submanifolds of indefinite Kaehler manifolds. A special class of CR-lightlike submanifolds is the class of CR-lightlike product submanifolds. Duggal and Bejancu [5] and Kumar et al. [6] characterized a CRlightlike submanifold to be a CR-lightlike product. In [7], the notion of warped product manifolds was introduced by Bishop and O' Neill in 1969 and it was studied by many mathematicians and physicists. These manifolds are generalization of Riemannian product manifolds. This generalized product metric appears in differential geometric studies in a natural way. For instance, a surface of revolution is a warped product manifold. Moreover, many important submanifolds in real and complex space forms are expressed as warped product submanifolds. In view of its physical applications, many research articles have recently appeared exploring existence (or nonexistence) of warped product submanifolds in known spaces (cf. [8,9], etc.). Chen [10] introduced warped product CR-submanifolds 
and showed that there does not exist a warped product CR-submanifold in the form $M=N_{\perp} \times_{\lambda} N_{T}$ in a Kaehler manifold where $N_{\perp}$ is a totally real submanifold and $N_{T}$ is a holomorphic submanifold of $\bar{M}$. He proved if $M=N_{\perp} \times_{\curlywedge} N_{T}$ is a warped product CR-submanifold of a Kaehler manifold $\bar{M}$, then $M$ is a CR-product, that is, there do not exist warped product CR-submanifolds of the form $M=N_{\perp} \times_{\lambda} N_{T}$ other than CR-product. Therefore, he called a warped product CR-submanifold in the form $M=N_{T} \times{ }_{\lambda} N_{\perp}$ a CRwarped product. Chen also obtained a characterization for CR-submanifold of a Kaehler manifold to be locally a warped product submanifold. He showed that a CR-submanifold $M$ of a Kaehler manifold $\bar{M}$ is a CR-warped product if and only if $A_{J Z} X=J X(\mu) Z$ for each $X \in \Gamma(D), Z \in \Gamma\left(D^{\prime}\right), \mu$ a $C^{\infty}$-function on $M$ such that $Z \mu=0$ for all $Z \in \Gamma\left(D^{\prime}\right)$.

The growing importance of lightlike submanifolds and hypersurfaces in mathematical physics, especially in relativity, motivated us to club the concept of CR-warped product with lightlike geometry. In this paper, we showed that there does not exist a warped product CR-lightlike submanifold in the form $M=N_{\perp} \times_{\lambda} N_{T}$ other than CR-lightlike product in an indefinite Kaehler manifold. We also obtained some characterizations for a CR-lightlike submanifold to be locally a CR-lightlike warped product.

\section{Lightlike Submanifolds}

We recall notations and fundamental equations for lightlike submanifolds, which are due to [5] by Duggal and Bejancu.

Let $(\bar{M}, \bar{g})$ be a real $(m+n)$-dimensional semi-Riemannian manifold of constant index $q$ such that $m, n \geq 1,1 \leq q \leq m+n-1$ and let $(M, g)$ be an $m$-dimensional submanifold of $\bar{M}$ and $g$ the induced metric of $\bar{g}$ on $M$. If $\bar{g}$ is degenerate on the tangent bundle TM of $M$, then $M$ is called a lightlike submanifold of $\bar{M}$. For a degenerate metric $g$ on $M$,

$$
T M^{\perp}=\cup\left\{u \in T_{x} \bar{M}: \bar{g}(u, v)=0, \forall v \in T_{x} M, x \in M\right\}
$$

is a degenerate $n$-dimensional subspace of $T_{x} \bar{M}$. Thus, both $T_{x} M$ and $T_{x} M^{\perp}$ are degenerate orthogonal subspaces but no longer complementary. In this case, there exists a subspace $\operatorname{Rad} T_{x} M=T_{x} M \cap T_{x} M^{\perp}$ which is known as radical (null) subspace. If the mapping

$$
\operatorname{Rad} T M: x \in M \longrightarrow \operatorname{Rad} T_{x} M
$$

defines a smooth distribution on $M$ of rank $r>0$, then the submanifold $M$ of $\bar{M}$ is called $r$-lightlike submanifold and $\operatorname{Rad} T M$ is called the radical distribution on $M$.

Let $S(T M)$ be a screen distribution which is a semi-Riemannian complementary distribution of $\operatorname{Rad}(T M)$ in $T M$, that is,

$$
T M=\operatorname{Rad} T M \perp S(T M),
$$


$S\left(T M^{\perp}\right)$ is a complementary vector subbundle to $\operatorname{Rad} T M$ in $T M^{\perp}$. Let $\operatorname{tr}(T M)$ and $\operatorname{ltr}(T M)$ be complementary (but not orthogonal) vector bundles to $T M$ in $\left.T \bar{M}\right|_{M}$ and to $\operatorname{Rad} T M$ in $S\left(T M^{\perp}\right)^{\perp}$, respectively. Then, we have

$$
\begin{gathered}
\operatorname{tr}(T M)=\operatorname{ltr}(T M) \perp S\left(T M^{\perp}\right), \\
\left.T \bar{M}\right|_{M}=T M \oplus \operatorname{tr}(T M)=(\operatorname{Rad} T M \oplus \operatorname{ltr}(T M)) \perp S(T M) \perp S\left(T M^{\perp}\right) .
\end{gathered}
$$

Let $u$ be a local coordinate neighborhood of $M$ and consider the local quasiorthonormal fields of frames of $\bar{M}$ along $M$, on $u$ as $\left\{\xi_{1}, \ldots, \xi_{r}, W_{r+1}, \ldots, W_{n}, N_{1}, \ldots, N_{r}, X_{r+1}, \ldots, X_{m}\right\}$, where $\left\{\xi_{1}, \ldots, \xi_{r}\right\},\left\{N_{1}, \ldots, N_{r}\right\}$ are local lightlike bases of $\Gamma\left(\left.\operatorname{Rad} T M\right|_{u}\right), \Gamma\left(\left.\operatorname{ltr}(T M)\right|_{u}\right)$ and $\left\{W_{r+1}, \ldots, W_{n}\right\},\left\{X_{r+1}, \ldots, X_{m}\right\}$ are local orthonormal bases of $\Gamma\left(\left.S\left(T M^{\perp}\right)\right|_{u}\right)$ and $\Gamma\left(\left.S(T M)\right|_{u}\right)$, respectively. For this quasiorthonormal fields of frames, we have the following theorem.

Theorem 2.1 (see [5]). Let $\left(M, g, S(T M), S\left(T M^{\perp}\right)\right)$ be an r-lightlike submanifold of a semiRiemannian manifold $(\bar{M}, \bar{g})$. Then, there exists a complementary vector bundle $\operatorname{lt}(T M)$ of $\operatorname{Rad} T M$ in $S\left(T M^{\perp}\right)^{\perp}$ and a basis of $\Gamma\left(\left.\operatorname{ltr}(T M)\right|_{u}\right)$ consisting of smooth section $\left\{N_{i}\right\}$ of $\left.S\left(T M^{\perp}\right)^{\perp}\right|_{u^{\prime}}$ where $u$ is a coordinate neighborhood of $M$, such that

$$
\bar{g}\left(N_{i}, \xi_{j}\right)=\delta_{i j}, \quad \bar{g}\left(N_{i}, N_{j}\right)=0,
$$

where $\left\{\xi_{1}, \ldots, \xi_{r}\right\}$ is a lightlike basis of $\Gamma(\operatorname{Rad}(T M))$.

Let $\bar{\nabla}$ be the Levi-Civita connection on $\bar{M}$. Then, according to the decomposition (2.5), the Gauss and Weingarten formulas are given by

$$
\begin{gathered}
\bar{\nabla}_{X} Y=\nabla_{X} Y+h(X, Y), \quad \forall X, Y \in \Gamma(T M), \\
\bar{\nabla}_{X} U=-A_{U} X+\nabla_{X}^{\perp} U, \quad \forall X \in \Gamma(T M), U \in \Gamma(\operatorname{tr}(T M)),
\end{gathered}
$$

where $\left\{\nabla_{X} Y, A_{U} X\right\}$ and $\left\{h(X, Y), \nabla_{X}^{\perp} U\right\}$ belong to $\Gamma(T M)$ and $\Gamma(\operatorname{tr}(T M))$, respectively. Here, $\nabla$ is a torsion-free linear connection on $M, h$ is a symmetric bilinear form on $\Gamma(T M)$ which is called second fundamental form, and $A_{U}$ is a linear operator on $M$ and known as shape operator.

According to (2.4), considering the projection morphisms $L$ and $S$ of $\operatorname{tr}(T M)$ on $\operatorname{ltr}(T M)$ and $S\left(T M^{\perp}\right)$, respectively, (2.7) and (2.8) give

$$
\begin{gathered}
\bar{\nabla}_{X} Y=\nabla_{X} Y+h^{l}(X, Y)+h^{s}(X, Y), \\
\bar{\nabla}_{X} U=-A_{U} X+D_{X}^{l} U+D_{X}^{s} U,
\end{gathered}
$$

where we put $h^{l}(X, Y)=L(h(X, Y)), h^{s}(X, Y)=S(h(X, Y)), D_{X}^{l} U=L\left(\nabla_{X}^{\perp} U\right), D_{X}^{s} U=$ $S\left(\nabla_{X}^{\perp} U\right)$. 
As $h^{l}$ and $h^{s}$ are $\Gamma(\operatorname{ltr}(T M))$-valued and $\Gamma\left(S\left(T M^{\perp}\right)\right)$-valued, respectively, therefore, they are called the lightlike second fundamental form and the screen second fundamental form on $M$. In particular,

$$
\begin{aligned}
& \bar{\nabla}_{X} N=-A_{N} X+\nabla_{X}^{l} N+D^{s}(X, N), \\
& \bar{\nabla}_{X} W=-A_{W} X+\nabla_{X}^{s} W+D^{l}(X, W),
\end{aligned}
$$

where $X \in \Gamma(T M), N \in \Gamma(\operatorname{ltr}(T M))$, and $W \in \Gamma\left(S\left(T M^{\perp}\right)\right)$.

Using (2.4)-(2.5) and (2.9)-(2.12), we obtain

$$
\begin{gathered}
\bar{g}\left(h^{s}(X, Y), W\right)+\bar{g}\left(Y, D^{l}(X, W)\right)=g\left(A_{W} X, Y\right) \\
\bar{g}\left(h^{l}(X, Y), \xi\right)+\bar{g}\left(Y, h^{l}(X, \xi)\right)+g\left(Y, \nabla_{X} \xi\right)=0, \\
\bar{g}\left(A_{N} X, N^{\prime}\right)+\bar{g}\left(N, A_{N^{\prime}} X\right)=0
\end{gathered}
$$

for any $\xi \in \Gamma(\operatorname{Rad} T M), W \in \Gamma\left(S\left(T M^{\perp}\right)\right)$, and $N, N^{\prime} \in \Gamma(\operatorname{ltr}(T M))$.

Let $\bar{P}$ be a projection of $T M$ on $S(T M)$. Now, we consider the decomposition (2.3), we can write

$$
\begin{gathered}
\nabla_{X} \bar{P} Y=\nabla_{X}^{*} \bar{P} Y+h^{*}(X, \bar{P} Y) \\
\nabla_{X} \xi=-A_{\xi}^{*} X+\nabla_{X}^{* t} \xi
\end{gathered}
$$

for any $X, Y \in \Gamma(T M)$, and $\xi \in \Gamma(\operatorname{Rad} T M)$, where $\left\{\nabla_{X}^{*} \bar{P} Y, A_{\xi}^{*} X\right\}$ and $\left\{h^{*}(X, \bar{P} Y), \nabla_{X}^{* t} \xi\right\}$ belong to $\Gamma(S(T M))$ and $\Gamma(\operatorname{Rad} T M)$, respectively. Here $\nabla^{*}$ and $\nabla_{X}^{* t}$ are linear connections on $S(T M)$ and $\operatorname{Rad} T M$, respectively. By using (2.9)-(2.10) and (2.16), we obtain

$$
\begin{aligned}
\bar{g}\left(h^{l}(X, \bar{P} Y), \xi\right) & =g\left(A_{\xi}^{*} X, \bar{P} Y\right), \\
\bar{g}\left(h^{*}(X, \bar{P} Y), N\right) & =\bar{g}\left(A_{N} X, \bar{P} Y\right) .
\end{aligned}
$$

Definition 2.2. Let $(\bar{M}, \bar{J}, \bar{g})$ be a real $2 m$-dimensional indefinite Kaehler manifold and let $M$ be an $n$-dimensional submanifold of $\bar{M}$. Then $M$ is said to be a CR-lightlike submanifold if the following two conditions are fulfilled:

(a) $J(\operatorname{Rad} T M)$ is distribution on $M$ such that

$$
\operatorname{Rad} T M \cap J(\operatorname{Rad} T M)=0 ;
$$

(b) there exist vector bundles $S(T M), S\left(T M^{\perp}\right), \operatorname{ltr}(T M), D_{0}$ and $D^{\prime}$ over $M$, such that

$$
S(T M)=\left\{J(\operatorname{Rad} T M) \oplus D^{\prime}\right\} \perp D_{0} ; \quad J\left(D_{0}\right)=D_{0} ; \quad J\left(D^{\prime}\right)=L_{1} \perp L_{2},
$$


where $\Gamma\left(D_{0}\right)$ is a nondegenerate distribution on $M, \Gamma\left(L_{1}\right)$ and $\Gamma\left(L_{2}\right)$ are vector subbundles of $\Gamma(\operatorname{ltr}(T M))$ and $\Gamma\left(S\left(T M^{\perp}\right)\right)$, respectively, and assume that $M_{1}=J\left(L_{1}\right)$ and $M_{2}=J\left(L_{2}\right)$.

Clearly, the tangent bundle of a CR-lightlike submanifold is decomposed as

$$
T M=D \oplus D^{\prime},
$$

where

$$
D=\operatorname{Rad} T M \perp J(\operatorname{Rad} T M) \perp D_{0} .
$$

Now, let $S$ and $Q$ be the projections on $D$ and $D^{\prime}$, respectively. Then, for any $X \in$ $\Gamma(T M)$, we can write

$$
X=S X+Q X
$$

where $S X \in D$ and $Q X \in D^{\prime}$. Applying $J$ to above equation, we get

$$
J X=f X+w X,
$$

where $f X=\bar{J} S X$ and $w X=\bar{J} Q X$. Clearly $f$ is a tensor field of type $(1,1)$ and $w$ is $\Gamma\left(L_{1} \perp L_{2}\right)$ valued 1-form on $M$. Clearly, $X \in \Gamma(D)$ if and only if $w X=0$. On the other hand, we set

$$
J V=B V+C V,
$$

for any $V \in \Gamma(\operatorname{tr}(T M))$, where $B V$ and $C V$ are sections of $T M$ and $\operatorname{tr}(T M)$, respectively.

By using Kaehlerian property of $\bar{\nabla}$ with (2.7) and (2.8), we have the following lemmas.

Lemma 2.3. Let $M$ be a CR-lightlike submanifold of an indefinite Kaehler manifold $\bar{M}$ then, one has

$$
\begin{gathered}
\left(\nabla_{X} f\right) Y=A_{w Y} X+B h(X, Y), \\
\left(\nabla_{X}^{t} w\right) Y=C h(X, Y)-h(X, f Y),
\end{gathered}
$$

for any $X, Y \in \Gamma(T M)$, where

$$
\begin{aligned}
& \left(\nabla_{X} f\right) Y=\nabla_{X} f Y-f\left(\nabla_{X} Y\right) \\
& \left(\nabla_{X}^{t} w\right) Y=\nabla_{X}^{t} w Y-w\left(\nabla_{X} Y\right)
\end{aligned}
$$


Lemma 2.4. Let $M$ be a $C R$-lightlike submanifold of an indefinite Kaehler manifold $\bar{M}$ then, one has

$$
\begin{gathered}
\left(\nabla_{X} B\right) V=-f A_{V} X+A_{C V} X, \\
\left(\nabla_{X} C\right) V=-w A_{V} X-h(X, B V),
\end{gathered}
$$

for any $X \in \Gamma(T M)$ and $V \in \Gamma(\operatorname{tr}(T M))$, where

$$
\begin{aligned}
& \left(\nabla_{X} B\right) V=\nabla_{X} B V-B \nabla_{X}^{t} V, \\
& \left(\nabla_{X} C\right) V=\nabla_{X}^{t} C V-C \nabla_{X}^{t} V .
\end{aligned}
$$

Theorem 2.5 (see [5]). Let $M$ be a CR-lightlike submanifold of an indefinite Kaehler manifold $\bar{M}$. Then, one has the following assertions.

(i) The almost complex distribution $D$ is integrable if and only if the second fundamental form of $M$ satisfies

$$
h(X, J Y)=h(J X, Y), \quad \forall X, Y \in \Gamma(D)
$$

(ii) The totally real distribution $D^{\prime}$ is integrable if and only if the shape operator of $M$ satisfies

$$
A_{J Z} U=A_{J U} Z, \quad \forall Z, U \in \Gamma\left(D^{\prime}\right)
$$

Theorem 2.6 (see [5]). Let $M$ be a CR-lightlike submanifold of an indefinite Kaehler manifold $\bar{M}$. Then, $D$ defines a totally geodesic foliation on $M$ if and only if, for any $X, Y \in \Gamma(D), h(X, Y)$ has no component in $\Gamma\left(L_{1} \perp L_{2}\right)$.

\section{CR-Lightlike Warped Product}

\section{Warped Product}

Let $B$ and $F$ be two Riemannian manifolds with Riemannian metrics $g_{B}$ and $g_{F}$, respectively, and $\lambda>0$ a differentiable function on $B$. Assume the product manifold $B \times F$ with its projection $\pi: B \times F \rightarrow B$ and $\eta: B \times F \rightarrow F$. The warped product $M=B \times{ }_{\lambda} F$ is the manifold $B \times F$ equipped with the Riemannian metric $g$, where

$$
g=g_{B}+\lambda^{2} g_{F}
$$

If $X$ is tangent to $M=B \times_{\lambda} F$ at $(p, q)$, then using (3.1), we have

$$
\|X\|^{2}=\left\|\pi_{*} X\right\|^{2}+\lambda^{2}(\pi(X))\left\|\eta_{*} X\right\|^{2}
$$


The function $\lambda$ is called the warping function of the warped product. For differentiable function $\lambda$ on $M$, the gradient $\nabla \lambda$ is defined by $g(\nabla \lambda, X)=X \lambda$, for all $X \in T(M)$.

Lemma 3.1 (see [7]). Let $M=B \times_{\curlywedge} F$ be a warped product manifold. If $X, Y \in T(B)$ and $U, V \in$ $T(F)$, then

$$
\begin{gathered}
\nabla_{X} Y \in T(B), \\
\nabla_{X} V=\nabla_{V} X=\frac{X \lambda}{\lambda} V, \\
\nabla_{U} V=-\frac{g(U, V)}{\lambda} \nabla \lambda .
\end{gathered}
$$

Corollary 3.2. On a warped product manifold $M=B \times_{\mathfrak{\lambda}}$ F one has

(i) $B$ is totally geodesic in $M$,

(ii) $F$ is totally umbilical in $M$.

Definition 3.3 (see [11]). A lightlike submanifold $(M, g)$ of a semi-Riemannian manifold $(\bar{M}, \bar{g})$ is said to be totally umbilical in $\bar{M}$ if there is a smooth transversal vector field $H \in \Gamma(\operatorname{tr}(T M))$ on $M$, called the transversal curvature vector field of $M$, such that

$$
h(X, Y)=H g(X, Y), \quad \forall X, Y \in \Gamma(T M)
$$

it is easy to see that $M$ is a totally umbilical if and only if on each coordinate neighborhood $u$, there exist smooth vector fields $H^{l} \in \Gamma(\operatorname{ltr}(T M))$ and $H^{s} \in \Gamma\left(S\left(T M^{\perp}\right)\right)$, such that

$$
h^{l}(X, Y)=H^{l} g(X, Y), \quad h^{s}(X, Y)=H^{s} g(X, Y), \quad D^{l}(X, W)=0,
$$

for any $W \in \Gamma\left(S\left(T M^{\perp}\right)\right)$.

Lemma 3.4. Let $M$ be a totally umbilical CR-lightlike submanifold of an indefinite Kaehler manifold $\bar{M}$ then, the distribution $D^{\prime}$ defines a totally geodesic foliation in $M$.

Proof. Let $X, Y \in \Gamma\left(D^{\prime}\right)$, then (2.25) and (2.27) imply that $f \nabla_{X} Y=-A_{w} X-B h(X, Y)$. Let $Z \in \Gamma\left(D_{0}\right)$, then

$$
\begin{aligned}
g\left(f \nabla_{X} Y, Z\right) & =-g\left(A_{w Y} X, Z\right)=\bar{g}\left(\bar{\nabla}_{X} J Y, Z\right) \\
& =-\bar{g}\left(\bar{\nabla}_{X} Y, J Z\right)=-\bar{g}\left(\bar{\nabla}_{X} Y, Z^{\prime}\right)=g\left(Y, \nabla_{X} Z^{\prime}\right)
\end{aligned}
$$

where, $Z^{\prime}=J Z \in \Gamma\left(D_{0}\right)$. Since $X \in \Gamma\left(D^{\prime}\right)$ and $Z \in \Gamma\left(D_{0}\right)$ then (2.26) and (2.28) imply that $w \nabla_{X} Z=h(X, f Z)-C h(X, Z)=H g(X, f Z)-C H g(X, Z)=0$, this implies that $\nabla_{X} Z \in \Gamma(D)$, then (3.8) implies that $g\left(f \nabla_{X} Y, Z\right)=0$, then the nondegeneracy of the distribution $D_{0}$ implies that $f \nabla_{X} Y=0$ gives $\nabla_{X} Y \in \Gamma\left(D^{\prime}\right)$ for any $X, Y \in \Gamma\left(D^{\prime}\right)$. Hence, the proof is complete. 
Theorem 3.5. Let $M$ be a totally umbilical CR-lightlike submanifold of an indefinite Kaehler manifold, then the totally real distribution $D^{\prime}$ is integrable.

Proof. Using (2.25) and (2.27) with the above lemma, for any $X, Y \in \Gamma\left(D^{\prime}\right)$, we get

$$
A_{w} X=-B h(X, Y)
$$

this implies $A_{w Y} X \in \Gamma\left(D^{\prime}\right)$ and also

$$
A_{w X} Y=-B h(Y, X)
$$

therefore, using (3.9) and (3.10), we get $A_{w \Upsilon} X=A_{w X} Y$, for any $X, Y \in \Gamma\left(D^{\prime}\right)$. This implies that the distribution $D^{\prime}$ is integrable.

Definition 3.6 (see [5]). A CR-lightlike submanifold $M$ of an indefinite Kaehler manifold $\bar{M}$ is called a CR-lightlike product if both the distribution $D$ and $D^{\prime}$ define totally geodesic foliations in $M$.

Theorem 3.7. Let $M$ be a totally umbilical CR-lightlike submanifold $M$ of an indefinite Kaehler manifold $\bar{M}$. If $M=N_{\perp} \times_{\curlywedge} N_{T}$ be a warped product CR-lightlike submanifold, then it is a CRlightlike product.

Proof. Since $M$ is a totally umbilical CR-lightlike submanifold of an indefinite Kaehler manifold, then using Lemma 3.4, the distribution $D^{\prime}$ defines a totally geodesic foliation in M.

Let $h^{T}$ and $A^{T}$ be the second fundamental form and the shape operator of $N_{T}$ in $M$, then for $X, Y \in \Gamma(D)$ and $Z \in \Gamma\left(D^{\prime}\right)$, we have $g\left(h^{T}(X, Y), Z\right)=g\left(\nabla_{X} Y, Z\right)=-\bar{g}\left(Y, \bar{\nabla}_{X} Z\right)=$ $-g\left(Y, \nabla_{X} Z\right)$. Using (3.4), we get

$$
g\left(h^{T}(X, Y), Z\right)=-(Z \ln \lambda) g(X, Y)
$$

Now, let $\widehat{h}$ be the second fundamental form of $N_{T}$ in $\bar{M}$, then

$$
\widehat{h}(X, Y)=h^{T}(X, Y)+h^{S}(X, Y)+h^{l}(X, Y)
$$

for any $X, Y$ tangent to $N_{T}$, then using (3.11), we get

$$
g(\widehat{h}(X, Y), Z)=g\left(h^{T}(X, Y), Z\right)=-(Z \ln \lambda) g(X, Y)
$$

Since $N_{T}$ is a holomorphic submanifold of $\bar{M}$, then we have $\widehat{h}(X, J Y)=\widehat{h}(J X, Y)=J \widehat{h}(X, Y)$, therefore, we have

$$
g(\widehat{h}(X, Y), Z)=-g(\widehat{h}(J X, J Y), Z)=(Z \ln \lambda) g(X, Y)
$$


Adding (3.13) and (3.14), we get

$$
g(\widehat{h}(X, Y), Z)=0
$$

Using (3.12), we have $g(h(X, Y), J Z)=g(\widehat{h}(X, Y), J Z)-g\left(h^{T}(X, Y), J Z\right)=g(\widehat{h}(X, Y), J Z)=$ $-g(J \widehat{h}(X, Y), Z)=-g(\widehat{h}(X, J Y), Z)=0$. Thus, $g(h(X, Y), J Z)=0$ implies that $h(X, Y)$ has no components in $L_{1} \perp L_{2}$ for any $X, Y \in \Gamma(D)$. This implies that the distribution $D$ defines a totally geodesic foliation in $M$. Hence, $M$ is a CR-lightlike product.

Theorem 3.7 shows that if $M=N_{\perp} \times_{\lambda} N_{T}$ is a warped product CR-lightlike submanifold of an indefinite Kaehler manifold, then it is CR-lightlike product, that is, there does not exist warped product CR-lightlike submanifolds of the form $M=N_{\perp} \times_{\lambda} N_{T}$ other than CR-lightlike product. Thus, for simplicity, we call a warped product CR-lightlike submanifold in the form $M=N_{T} \times_{\lambda} N_{\perp}$ a CR-lightlike warped product.

Lemma 3.8. Let $M$ be a totally umbilical $C R$-lightlike submanifold of an indefinite Kaehler manifold $\bar{M}$. Let $M=N_{T} \times_{\curlywedge} N_{\perp}$ be a proper CR-lightlike warped product of an indefinite Kaehler manifold, then $N_{T}$ is totally geodesic in $M$.

Proof. Let $\nabla$ be a linear connection on $M$ induced from $\bar{\nabla}$. Let $X, Y \in N_{T}$ and $Z \in N_{\perp}$, then we have $g\left(\nabla_{X} Y, Z\right)=\bar{g}\left(\bar{\nabla}_{X} Y, Z\right)=-g\left(Y, \nabla_{X} Z\right)-g\left(Y, h^{l}(X, Z)\right)$, using (3.4), we get $g\left(\nabla_{X} Y, Z\right)=-g\left(Y, h^{l}(X, Z)\right)$. Since $M$ is totally umbilical CR-lightlike submanifold, therefore, $h^{l}(X, Z)=h^{s}(X, Z)=0$. Hence, $g\left(\nabla_{X} Y, Z\right)=0$ implies that $N_{T}$ is totally geodesic in $M$.

Theorem 3.9 (see[6]). Let $M$ be a CR-lightlike submanifold of an indefinite Kaehler manifold $\bar{M}$. Then distribution $D$ defines totally geodesic foliation if and only if $D$ is integrable.

Theorem 3.10. Let $M$ be a totally umbilical proper CR-lightlike submanifold of an indefinite Kaehler manifold $\bar{M}$, then $H^{l}=0$.

Proof. Let $M$ be a totally umbilical proper CR-lightlike submanifold then using (2.25) and (2.27), we have $A_{w Z} Z=-f \nabla_{Z} Z-B h^{l}(Z, Z)-B h^{s}(Z, Z)$, for $Z \in \Gamma\left(M_{2}\right)$. We obtain $g\left(A_{J Z} Z, J \xi\right)+g\left(h^{l}(Z, Z), \xi\right)=0$. Using $(2.13)$ and the hypothesis we obtain $g(Z, Z) g\left(H^{l}, \xi\right)=$ 0 , then using the non degeneracy of $M_{2}$, the result follows.

\section{A Characterization of CR-Lightlike Warped Products}

For a CR-lightlike warped products in indefinite Kaehler manifolds, we have

Lemma 4.1. Let $M$ be a totally umbilical CR-lightlike submanifold of an indefinite Kaehler manifold $\bar{M}$, then for a CR-lightlike warped product $M=N_{T} \times{ }_{\lambda} N_{\perp}$ in an indefinite Kaehler manifold $\bar{M}$, one has

(i) $\bar{g}\left(h^{s}(D, D), J M_{2}\right)=0$,

(ii) $\bar{g}\left(h(J X, Z), J Z_{1}\right)=(X \ln \lambda) g\left(Z, Z_{1}\right)$,

for any $X \in \Gamma(D)$ and $Z, Z_{1} \in \Gamma\left(M_{2}\right) \subset \Gamma\left(D^{\prime}\right)$. 
Proof. Since $\bar{M}$ is Kaehlerian, therefore, for $X \in \Gamma(D)$ and $Z \in \Gamma\left(M_{2}\right)$, we have $J \bar{\nabla}_{X} Z=\bar{\nabla}_{X} J Z$, since $M$ is totally umbilical, therefore, we have $J\left(\nabla_{X} Z\right)=-A_{w Z} X+\nabla_{X}^{S} w Z$, then taking inner product with $J Y$, where $Y \in \Gamma(D)$, we get $g\left(\nabla_{X} Z, Y\right)=-g\left(A_{w Z} X, J Y\right)$. Using (3.4), we obtain $g\left(A_{w Z} X, J Y\right)=0$, then using (2.13), we get $\bar{g}\left(h^{S}(D, D), J M_{2}\right)=0$.

Next for any $X \in \Gamma(D)$ and $Z, Z_{1} \in \Gamma\left(M_{2}\right) \subset \Gamma\left(D^{\prime}\right)$, we have $g\left(h(J X, Z), J Z_{1}\right)=$ $g\left(\bar{\nabla}_{Z} J X, J Z_{1}\right)=g\left(\nabla_{Z} X, Z_{1}\right)=(X \ln \lambda) g\left(Z, Z_{1}\right)$. Hence, the proof is complete.

Corollary 4.2. Let $Z \in \Gamma\left(M_{1}\right) \subset \Gamma\left(D^{\prime}\right)$, then clearly $g\left(h^{s}(D, D), J Z\right)=0$ and also $g\left(h^{l}(D, D), J Z\right)=0$ for any $Z \in \Gamma\left(D^{\prime}\right)$. Thus, $g\left(h(D, D), J D^{\prime}\right)=0$, this implies that $h(D, D)$ has no component in $L_{1} \perp L_{2}$, therefore, using Theorem 2.5 , the distribution $D$ defines a totally geodesic foliation in $M$.

We have the following some characterizations of CR-lightlike warped product.

Theorem 4.3. A proper totally umbilical CR-lightlike submanifold $M$ of an indefinite Kaehler manifold $\bar{M}$ is locally a CR-lightlike warped product if and only if

$$
A_{J Z} X=((J X) \mu) Z,
$$

for $X \in D, Z \in D^{\prime}$ and for some function $\mu$ on $M$ satisfying $U \mu=0, U \in \Gamma\left(D^{\prime}\right)$.

Proof. Assume that $M$ be a proper CR-lightlike submanifold of an indefinite Kaehler manifold $\bar{M}$ satisfying (4.1). Let $Y \in \Gamma(D)$ and $Z \in \Gamma\left(M_{2}\right) \subset \Gamma\left(D^{\prime}\right)$, we have $g\left(A_{J Z} X, J Y\right)=$ $g(((J X) \mu) Z, J Y)=0$, then using (2.13), we get $g\left(h^{s}(D, D), J M_{2}\right)=0$. If $Z \in \Gamma\left(M_{1}\right) \subset \Gamma\left(D^{\prime}\right)$, then clearly $g\left(h^{s}(D, D), J Z\right)=0$ and also $g\left(h^{l}(D, D), J Z\right)=0$ for any $Z \in \Gamma\left(D^{\prime}\right)$. Thus,

$$
g\left(h(D, D), J D^{\prime}\right)=0,
$$

that is, $h(D, D)$ has no component in $L_{1} \perp L_{2}$, this implies that the distribution $D$ defines totally geodesic foliation in $M$ and consequently it is totally geodesic in $M$ and using Theorem 3.9, the distribution $D$ is integrable.

Taking inner product of (4.1) with $U \in \Gamma\left(D^{\prime}\right)$ and using that $M$ is totally umbilical, we get $g(((J X) \mu) Z, U)=g\left(A_{J Z} X, U\right)=g\left(J Z, \nabla_{X} U\right)=g\left(J Z, \nabla_{U} X\right)=-\bar{g}\left(\bar{\nabla}_{U} J Z, X\right)=$ $g\left(\nabla_{U} Z, J X\right)$, using the definition of gradient $g(\nabla \phi, X)=X \phi$, we get

$$
g\left(\nabla_{U} Z, J X\right)=g(\nabla \mu, J X) g(Z, U)
$$

Let $h^{\prime}$ be the second fundamental form of $D^{\prime}$ in $M$ and let $\nabla^{\prime}$ be the metric connection of $D^{\prime}$ in $M$ then, particularly for $X \in \Gamma\left(D_{0}\right)$, we have

$$
g\left(h^{\prime}(U, Z), J X\right)=g\left(\nabla_{U} Z-\nabla_{U}^{\prime} Z, J X\right)=g\left(\nabla_{U} Z, J X\right)
$$

Therefore, from (4.3) and (4.4), we get $g\left(h^{\prime}(U, Z), J X\right)=g(\nabla \mu, J X) g(Z, U)$, this further implies that

$$
h^{\prime}(U, Z)=\nabla \mu g(Z, U)
$$


this implies that the distribution $D^{\prime}$ is totally umbilical in $M$. Using Theorem 3.5, the totally real distribution $D^{\prime}$ is integrable and (4.5) and the condition $U \mu=0$ for $U \in D^{\prime}$ imply that each leaf of $D^{\prime}$ is an extrinsic sphere in $M$. Hence, by a result of [12] which say that "if the tangent bundle of a Riemannian manifold $M$ splits into an orthogonal sum $T M=E_{0} \oplus E_{1}$ of nontrivial vector subbundles such that $E_{1}$ is spherical and its orthogonal complement $E_{0}$ is autoparallel, then the manifold $M$ is locally isometric to a warped product $M_{0} \times_{\lambda} M_{1}, "$ " therefore, we can conclude that $M$ is locally a CR-lightlike warped product $N_{T} \times{ }_{\curlywedge} N_{\perp}$ of $\bar{M}$, where $\lambda=e^{\mu}$.

Conversely, let $X \in \Gamma\left(N_{T}\right)$ and $Z \in \Gamma\left(N_{\perp}\right)$, since $\bar{M}$ is a Kaehler manifold so, we have $\bar{\nabla}_{X} J Z=J \bar{\nabla}_{X} Z$, which further becomes $-A_{J Z} X+\nabla_{X}^{t} J Z=((J X) \ln \lambda) Z$, comparing tangential components, we get $A_{J Z} X=-((J X) \ln \lambda) Z$ for each $X \in \Gamma(D)$ and $Z \in\left(D^{\prime}\right)$. Since $\lambda$ is a function on $N_{T}$, so we also have $U(\ln \lambda)=0$ for all $U \in \Gamma\left(D^{\prime}\right)$. Hence, the proof is complete.

Lemma 4.4. Let $M=N_{T} \times_{\curlywedge} N_{\perp}$ be a CR-lightlike warped product of an indefinite Kaehler manifold $\bar{M}$, then

$$
\begin{gathered}
\left(\nabla_{Z} f\right) X=f X(\ln \lambda) Z . \\
\left(\nabla_{U} f\right) Z=g(Z, U) f(\nabla \ln \lambda),
\end{gathered}
$$

for any $U \in \Gamma(T M), X \in \Gamma\left(N_{T}\right)$, and $Z \in \Gamma\left(N_{\perp}\right)$.

Proof. For $X \in \Gamma\left(N_{T}\right)$ and $Z \in \Gamma\left(N_{\perp}\right)$, using (2.27) and (3.4), we have $\left(\nabla_{Z} f\right) X=\nabla_{Z} f X=$ $f X(\ln \lambda) Z$. Next, again using $(2.27)$, we get $\left(\nabla_{U} f\right) Z=-f \nabla_{U} Z$, this implies that $\left(\nabla_{U} f\right) Z \in$ $\Gamma\left(N_{T}\right)$, therefore, for any $X \in \Gamma\left(D_{0}\right)$, we have

$$
\begin{aligned}
g\left(\left(\nabla_{U} f\right) Z, X\right) & =-g\left(f \nabla_{U} Z, X\right)=g\left(\nabla_{U} Z, f X\right)=\bar{g}\left(\bar{\nabla}_{U} Z, f X\right) \\
& =-g\left(Z, \nabla_{U} f X\right)=-f X(\ln \lambda) g(Z, U) .
\end{aligned}
$$

Hence, using the definition of gradient of $\lambda$ and the nondegeneracy of the distribution $D_{0}$, the result follows.

Theorem 4.5. A proper totally umbilical CR-lightlike submanifold $M$ of an indefinite Kaehler manifold $\bar{M}$ is locally a CR-lightlike warped product if and only if

$$
\left(\nabla_{U} f\right) V=(f V(\mu)) Q U+g(Q U, Q V) J(\nabla \mu)
$$

for any $U, V \in \Gamma(T M)$ and for some function $\mu$ on $M$ satisfying $Z \mu=0, Z \in \Gamma\left(D^{\prime}\right)$.

Proof. Let $M$ be a CR-lightlike submanifold of an indefinite Kaehler manifold $\bar{M}$ satisfying (4.8). Let $U, V \in \Gamma(D)$, then (4.8) implies that $\left(\nabla_{U} f\right) V=0$, then (2.25) gives $B h(U, V)=0$. Thus $D$ defines a totally geodesic foliation in $M$ and consequently it is totally geodesic in $M$ and integrable using Theorem 3.9. 
Let $U, V \in \Gamma\left(D^{\prime}\right)$, then (4.8) gives

$$
\left(\nabla_{U} f\right) V=g(Q U, Q V) J(\nabla \mu) .
$$

Let $X \in \Gamma\left(D_{0}\right)$, then (4.9) implies that

$$
g\left(\left(\nabla_{U} f\right) V, X\right)=g(Q U, Q V) g(J(\nabla \mu), X) .
$$

Also

$$
g\left(\left(\nabla_{U} f\right) V, X\right)=g\left(A_{w V} U, X\right)=\bar{g}\left(\bar{\nabla}_{U} V, J X\right)=g\left(\nabla_{U} V, J X\right)
$$

therefore, from (4.10) and (4.11), we get

$$
g\left(\nabla_{U} V, J X\right)=-g(\nabla \mu, J X) g(U, V) .
$$

Let $h^{\prime}$ be the second fundamental form of $D^{\prime}$ in $M$ and let $\nabla^{\prime}$ be the metric connection of $D^{\prime}$ in $M$, then

$$
g\left(h^{\prime}(U, V), J X\right)=g\left(\nabla_{U} V, J X\right)
$$

therefore, from (4.12) and (4.13), we get $g\left(h^{\prime}(U, V), J X\right)=-g(\nabla \mu, J X) g(U, V)$, then the nondegeneracy of the distribution $D_{0}$ implies that

$$
h^{\prime}(U, V)=-\nabla \mu g(U, V) \text {, }
$$

this gives that the distribution $D^{\prime}$ is totally umbilical in $M$ and using Theorem 3.5, the distribution $D^{\prime}$ is integrable. Also, $Z \mu=0$ for $Z \in \Gamma\left(D^{\prime}\right)$, hence as in Theorem 4.3, each leaf of $D^{\prime}$ is an extrinsic sphere in $M$. Thus, $M$ is locally a CR-lightlike warped product $N_{T} \times_{\lambda} N_{\perp}$ of $\bar{M}$, where $\lambda=e^{\mu}$.

Conversely, let $M$ be a CR-lightlike warped product $N_{T} \times_{\lambda} N_{\perp}$ of an indefinite Kaehler manifold $\bar{M}$. Using (2.22), we can write

$$
\left(\nabla_{U} f\right) V=\left(\nabla_{S U} f\right) S V+\left(\nabla_{Q U} f\right) S V+\left(\nabla_{U} f\right) Q V .
$$

Since $D$ defines totally geodesic foliation in $M$, therefore, using (2.25), we get

$$
\left(\nabla_{S U} f\right) S V=0 .
$$

Using (4.6), we have

$$
\begin{gathered}
\left(\nabla_{Q U} f\right) S V=f V(\ln \lambda) Q U, \\
\left(\nabla_{U} f\right) Q V=g(Q U, Q V) f(\nabla \ln \lambda) .
\end{gathered}
$$

Hence, from (4.15)-(4.18), the result follows. 
Theorem 4.6. Let $M$ be a locally CR-lightlike warped product of an indefinite Kaehler manifold $\bar{M}$, then

$$
\bar{g}\left(\left(\nabla_{U}^{t} w\right) V, J Z\right)=-S V(\mu) g(U, Z),
$$

for any $U, V \in \Gamma(T M)$ and for some function $\mu$ on $M$ satisfying $Z \mu=0, Z \in \Gamma\left(D^{\prime}\right)$.

Proof. Let $M$ be a CR-lightlike warped product of an indefinite Kaehler manifold $\bar{M}$. Therefore, the distribution $D$ defines totally geodesic foliation in $M$, then using (2.25) for $U, V \in \Gamma(D)$, we get

$$
\begin{aligned}
\bar{g}\left(\left(\nabla_{U}^{t} w\right) V, J Z\right) & =-g(h(U, f V), J Z)=-g\left(\bar{\nabla}_{U} V, Z\right)+g\left(\nabla_{U} f V, J Z\right) \\
& =-g\left(\nabla_{U} V, Z\right)+g\left(f \nabla_{U} V, J Z\right)=0 .
\end{aligned}
$$

For $U \in \Gamma(D), V \in \Gamma\left(D^{\prime}\right)$ or $U, V \in \Gamma\left(D^{\prime}\right)$, using (2.25), we have

$$
\bar{g}\left(\left(\nabla_{U}^{t} w\right) V, J Z\right)=0
$$

Now let $U \in \Gamma\left(D^{\prime}\right)$ and $V \in \Gamma(D)$, then using (3.4), we have

$$
\begin{aligned}
\bar{g}\left(\left(\nabla_{U}^{t} w\right) V, J Z\right) & =-g(h(U, f V), J Z)=-g\left(\nabla_{U} V, Z\right)+g\left(f \nabla_{U} V, J Z\right) \\
& =-V(\ln \lambda) g(U, Z) .
\end{aligned}
$$

Therefore, (4.19) follows from (4.21)-(4.22). Hence, the result is complete.

\section{References}

[1] A. Bejancu, "CR submanifolds of a Kaehler manifold. II," Transactions of the American Mathematical Society, vol. 250, pp. 333-345, 1979.

[2] A. Bejancu, M. Kon, and K. Yano, "CR-submanifolds of a complex space form," Journal of Differential Geometry, vol. 16, no. 1, pp. 137-145, 1981.

[3] B. Y. Chen, "CR-submanifolds of a Kaehler manifold. I," Journal of Differential Geometry, vol. 16, no. 2, pp. 305-322, 1981.

[4] D. E. Blair and B. Y. Chen, “On CR-submanifolds of Hermitian manifolds," Israel Journal of Mathematics, vol. 34, no. 4, pp. 353-363, 1979.

[5] K. L. Duggal and A. Bejancu, Lightlike Submanifolds of Semi-Riemannian Manifolds and Applications, vol. 364 of Mathematics and its Applications, Kluwer Academic Publishers, Dordrecht, The Netherlands, 1996.

[6] R. Kumar, J. Kaur, and R. K. Nagaich, "On CR-lightlike product of an indefinite Kaehler manifold," International Journal of Mathematics and Mathematical Sciences, vol. 2011, Article ID 857953, 10 pages, 2011.

[7] R. L. Bishop and B. O'Neill, "Manifolds of negative curvature," Transactions of the American Mathematical Society, vol. 145, pp. 1-49, 1969.

[8] I. Hasegawa and I. Mihai, "Contact CR-warped product submanifolds in Sasakian manifolds," Geometriae Dedicata, vol. 102, pp. 143-150, 2003.

[9] B. Sahin, "Nonexistence of warped product semi-slant submanifolds of Kaehler manifolds," Geometriae Dedicata, vol. 117, pp. 195-202, 2006. 
[10] B. Y. Chen, "Geometry of warped product CR-submanifolds in Kaehler manifolds," Monatshefte für Mathematik, vol. 133, no. 3, pp. 177-195, 2001.

[11] K. L. Duggal and D. H. Jin, "Totally umbilical lightlike submanifolds," Kodai Mathematical Journal, vol. 26, no. 1, pp. 49-68, 2003.

[12] S. Hiepko, "Eine innere Kennzeichnung der verzerrten produkte," Mathematische Annalen, vol. 241, no. 3, pp. 209-215, 1979. 




Advances in

Operations Research

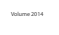



\section{The Scientific} World Journal
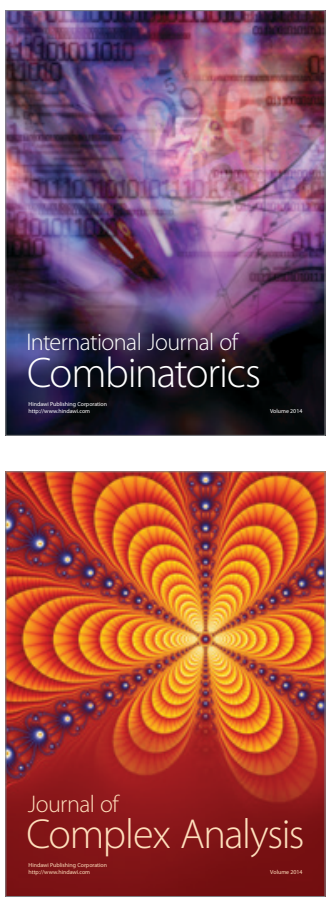

International Journal of

Mathematics and

Mathematical

Sciences
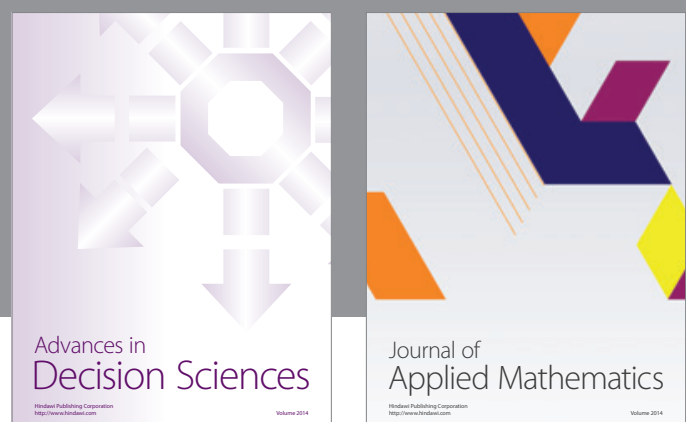

Journal of

Applied Mathematics
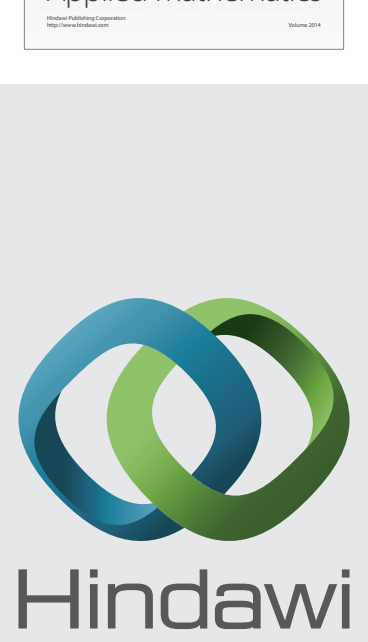

Submit your manuscripts at http://www.hindawi.com
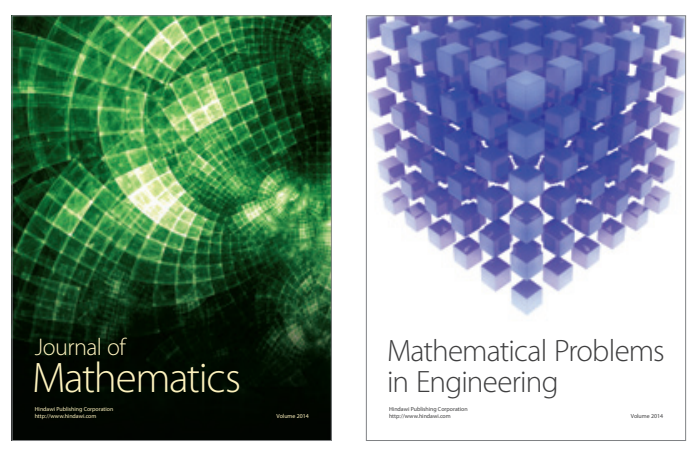

Mathematical Problems in Engineering


Journal of

Function Spaces
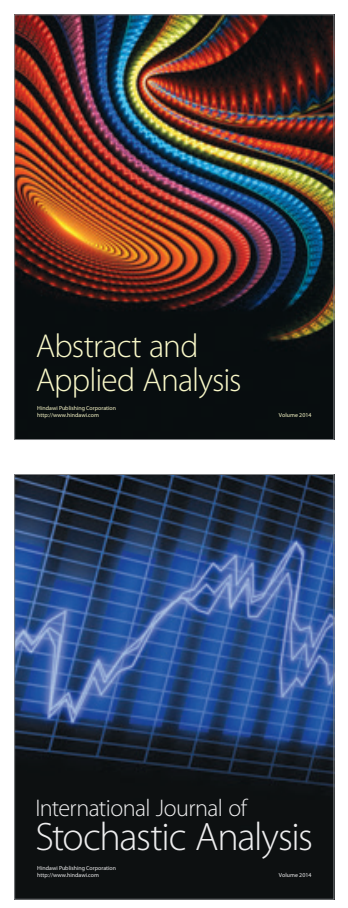

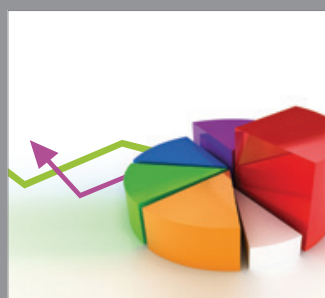

ournal of

Probability and Statistics

Promensencen
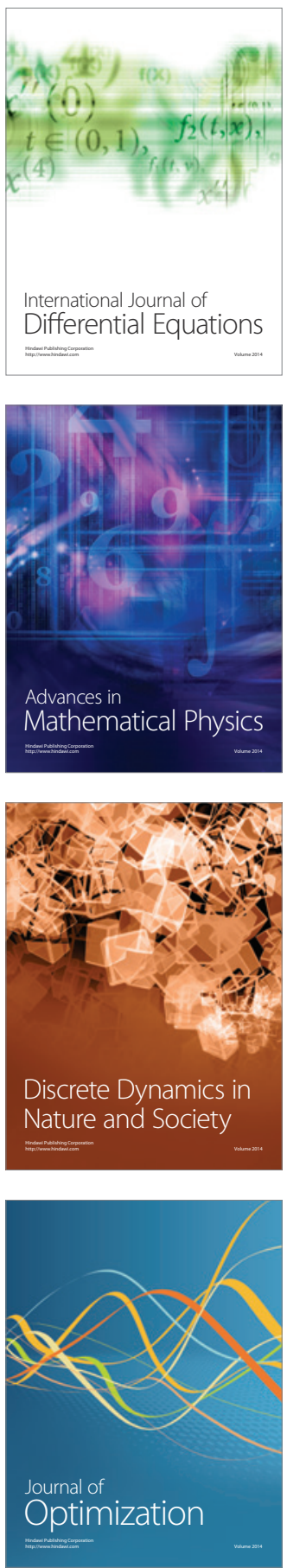